\title{
RADIAL NERVE PALSIES IN THE NEWBORN
}

\author{
BY \\ GEOFFREY V. FELDMAN \\ From the Department of Child Health, University of Manchester
}

(RECEIVED FOR PUBLICATION MAY 3, 1957)

\begin{abstract}
Although usually omitted in the description of nerve injuries in the newborn, radial nerve palsies are not infrequent. The cause of the paralysis is not clear but the occasional coincidental finding of a patch of subcutaneous fat necrosis over the course of the radial nerve (Lightwood, 1951) suggests the possibility of direct trauma to the nerve.

That the injury is not uncommon is supported by the observation of seven infants born in St. Mary's Hospital, Manchester, since 1953 with radial palsy, and an eighth seen by Professor Morris. In view of the scant literature on the subject it was considered worth while recording these cases.
\end{abstract}

\section{Case Reports}

Case 1. A primigravida aged 22 years was admitted in labour. Her pains lasted for seven days and a female infant (L.B.) was delivered by normal forces $45 \frac{1}{2}$ hours after rupture of the membranes.

During the second stage of the labour a diagnosis of hypertonic uterine inertia was made and it was thought that a constriction ring was palpable at one stage.

The infant weighed $7 \mathrm{lb}$. $14 \frac{1}{2} \mathrm{oz}$. and although no abnormality was detected at birth, on the fourth day a right-sided wrist drop was noted. On the sixth day a small patch of subcutaneous fat necrosis was detected over the lateral aspect of the right upper arm in a position overlying the radial nerve.

Four and a half weeks after birth, during which time a cock-up splint and passive movements had been the treatment, practically full movement had returned to the wrist.

Case 2. A primigravida aged 23 years had a lower segment caesarean section following a prolonged labour with cervical dystocia. During labour the uterus was said to be very tense and difficult to palpate.

The infant (J.D.), a boy weighing $7 \mathrm{lb} .13 \mathrm{oz}$., required resuscitation and regular respiration was not established for 10 minutes.

No abnormality was noted at birth but on the sixth day a left-sided wrist drop was detected and a ' cock-up' splint applied. Six weeks after birth normal movement was present in the wrist.

Case 3. A primigravida aged 31 years was delivered by lower segment caesarean section on account of uterine inertia after a first stage of over 80 hours and premature rupture of the membranes.

The infant (M.R.), who was a girl weighing $8 \mathrm{lb} .8 \mathrm{oz}$., exhibited only irregular gasping respiration for the first 10 minutes and subsequently showed evidence of atelectasis and 'cerebral signs'.

On the day after birth a right wrist drop, associated with an area of subcutaneous fat necrosis over the lateral aspect of the right upper arm, was present. The radial palsy had completely recovered eight days after birth.

Case 4. After labour lasting 48 hours and complicated only by an intrapartum pyrexia, a primigravid woman aged 24 years gave birth to a female infant (A.W.) weighing $8 \mathrm{lb}$. who breathed spontaneously.

The infant was thought to be postmature. She had bilateral wrist drop. Three days after birth subcutaneous fat necrosis was present on the lateral surface of both upper arms.

Function of both wrists had fully recovered three months after birth.

Case 5. A primiparous woman aged 23 years suffered from uterine inertia in both the first and second stages of labour. On this account lower forceps extraction was carried out and an $8 \mathrm{lb} .3 \mathrm{oz}$. male infant (J.C.) was delivered.

The infant was asphyxiated at birth but rapidly recovered. He was found to have a right facial palsy and bilateral wrist drop. On the inner aspect of both upper arms and at corresponding sites on the sides of the chest, pressure marks were visible. The day after delivery patches of subcutaneous fat necrosis appeared on the chest walls at the site of the pressure marks and in addition a further small patch appeared just above the lateral condyle of the right elbow. During the subsequent three days the left wrist drop progressively improved but the right side showed no sign of recovery and the area of subcutaneous fat necrosis increased in size.

By the twelfth day normal movement had returned to the left wrist but although there had been considerable improvement in the right wrist, recovery was not complete until five weeks after birth.

Case 6. A primipara aged 23 years had a delayed second stage of labour, the total duration of her labour being over 138 hours. She was finally delivered with 
the assistance of a low forceps extraction of a female infant (L.T.) weighing $6 \mathrm{lb} .12 \mathrm{oz}$.

The infant showed a large caput over the right brow with oedema of the eyelids on the right side. There was some evidence of atelectasis and bruising was noted over the middle of the lateral aspect of the left upper arm, a left wrist drop also being present. Subcutaneous fat necrosis developed on the arm at the site of the bruising. Five weeks after birth full movement had returned to the wrist and the fat necrosis was no longer detectable.

Case 7. A primigravida aged 30 years was delivered by normal forces of a female infant (J.M.) weighing $7 \mathrm{lb} .14 \mathrm{oz}$. A right-sided wrist drop and a patch of fat necrosis on the outer side of the right upper arm were noticed four days after birth, the left wrist also showing some weakness of extension. Three weeks after birth the wrists were moving satisfactorily.

Case 8. Professor W. I. C. Morris has kindly supplied the following description of a case of which he had care before coming to Manchester:

' In the spring of 1947 I attended, at the Western General Hospital, Edinburgh, a patient who was delivered by caesarean section of a living baby who showed a striking but temporary paralysis of the extensor muscles of its right wrist.

Labour was prolonged, lasting in all some 72 hours. The action of the uterus was irregular, but the extent of the disorder was not recognized until the third day, the house officer staff being lulled into a sense of false security by the fact that the membranes remained intact. The real facts of the situation were not recognized until I myself observed a slight sulcus which crossed the patient's abdominal wall at right angles to the long axis of the uterus at a level a little below the patient's umbilicus. This sulcus was constantly present. During active contraction of the uterus it became exaggerated, and a diagnosis of constriction ring was made. The infant was presenting by the head in the left occipitolateral position. The infant was well grown, but there was no disproportion, the head having already passed the plane of the pelvic brim.

It was decided to carry out a caesarean section. When the abdomen was opened, the sulcus was easily visible on the anterior uterine wall as an area of annular spasm, with a number of greatly dilated subperitoneal venules radiating from it both upwards and downwards in the long axis of the uterus. The uterus was opened with a transverse incision in the lower uterine segment. The infant's right shoulder presented through the uterine wound, and it was necessary to pass the hand deeply into the pelvis to disimpact the head before the baby could be extracted. As this was done, it was noted that the scalp was uniformly oedematous to a degree which gave rise to an erroneous suspicion that the infant might be suffering from hydrops foetalis. He was in fact quite normal, and once the head had been brought out through the uterine wound, he was extracted quite readily. He cried at once. The operation was completed in the usual manner. The mother made a good recovery and the baby, too, did well.
When the infant was examined after delivery, he was found to have a dropped wrist on the right side. The back was crossed obliquely by an area of ecchymosis, faint but quite definite, extending from a point in the posterior triangle of the neck on the left side downwards and towards the right. Over the right superior extremity there was a faint ecchymosis of the skin on the lateral side of the arm, corresponding to the line of ecchymosis on the infant's back, and the contour of the arm was quite noticeably concave, the ecchymosis being situated quite precisely over the middle of the shaft of the humerus immediately over the line of the musculospiral nerve. The infant's body on the cephalic side of the line of ecchymosis was quite markedly oedematous, the oedema involving the thoracic wall, the neck and the whole of the vertex.

It was concluded that pressure from the annular spasm of the uterus was responsible for the line of ecchymosis, and that the dropped wrist might be attributable to direct pressure of the constriction ring on the musculospiral nerve. The oedema of the foetus was thought likely to be due to interference with the venous drainage from the skin attributable to the same spasm.

The baby was photographed three hours after delivery. Twenty-four hours later the oedema had disappeared. The dropped wrist persisted for a few days, but had cleared up when the mother went home on the twentyfirst day.'

\section{Discussion}

In a review of the seven cases observed at St. Mary's Hospital, Manchester, no common factor, with the exception of the parity of the mother, comes to light. This could hardly be the direct cause of the injury.

However, with the exception of two cases, labour was prolonged and led to termination by abnormal means in four of these five cases, two by caesarean section and two by low forceps extraction. In two cases a diagnosis of hypertonic uterine inertia was made in both of which the presence of a constriction ring was suspected, and in a third the uterus was reported to be tense and difficult to palpate. That uterine inertia had occurred in a fourth case is known, though the tone of the uterus is not noted. Perhaps then Professor Morris's case may supply the clue to the causation of the radial palsy in these cases. There seems to be no doubt that from his description of the case the foetus had suffered pressure such as would be likely to cause trauma to the radial nerve. Could it then be that trauma of a similar type, though less obvious, has been the cause of the radial palsy in the four cases under review in which uterine inertia occurred? Certainly in Case 6 there had been sufficient intra-uterine pressure exerted to cause pressure marks and subsequent subcutaneous fat necrosis on the chest wall where the arms had been in contact. 
Even in the two cases in which there was no evidence of uterine inertia subcutaneous fat necrosis had occurred at sites overlying the course of the radial nerve, suggesting the possibility of pressure at this site. In one of these the fat necrosis and wrist drop were bilateral, a fact much more easily explained by a concentric pressure exerted by the uterus than from pressure by other means in the birth canal. Is it therefore not possible that a transient hypertonic state had existed in the uterus though not sufficiently long lasting to cause inertia or delay in delivery?

The prognosis for the radial palsy was good in all the cases mentioned though full recovery took a varying period of time, this prognosis being consistent with the cases reported by Lightwood. It is interesting to note that the labour in the first of Lightwood's cases was prolonged, though terminating by normal forces.

It is therefore suggested as a possible hypothesis to explain the occurrence of radial palsy in the newborn that some such cases may be due to abnormal uterine action, causing constriction of the foetus's arm at the site of the radial nerve.

\section{Summary}

Eight cases of radial palsy in the newborn are described. Uterine action was known to be abnormal in six of these cases. The prognosis for the radial nerve lesion was uniformly good.

It is suggested that a uterine constriction ring may be a cause of such radial nerve lesions.

My thanks are due to Professor Wilfrid Gaisford for his advice and for allowing me to include cases under his care; to Professor W. I. C. Morris for his help in supplying information regarding his case, and to Dr. George Komrower under whose care were some of the cases described.

REFERENCE

Lightwood, R. (1951) Archives of Disease in Childhood, 26, 436. 